\title{
Mast Cell-Derived Heparin Proteoglycans As a Model for a Local Antithrombotic
}

\author{
Riitta Lassila, MD, $\mathrm{PhD}^{1,2,3}$ Annukka Jouppila, $\mathrm{MSc}^{4}$ \\ ${ }^{1}$ Aplagon Ltd, Helsinki, Finland \\ ${ }^{2}$ Coagulation Disorders Unit, Hematology and Cancer Center, Helsinki \\ University Central Hospital, Helsinki, Finland \\ 3 Medical faculty, University of Helsinki, Helsinki, Finland \\ ${ }^{4}$ Coagulation Disorders Unit, Helsinki University Central Hospital \\ Research Institute, Helsinki, Finland
}

\begin{abstract}
Address for correspondence Riitta Lassila, MD, PhD, Coagulation Disorders Unit, Hematology and Cancer Center, Helsinki University Central Hospital, PoB 372, 00029 HUS, Helsinki, Finland (e-mail: riitta.lassila@hus.fi).
\end{abstract}

Semin Thromb Hemost 2014;40:837-844.

Abstract
Keywords
- heparin
- heparin proteoglycan
- antiplatelet
- anticoagulant

Mast cell-derived heparin proteoglycans (HEP-PG) reside in vascular tissue and serve as a local antithrombotic. Heparin, as used clinically, is isolated from its protein backbone from porcine and bovine gut mucosa. The isolated heparin is an anticoagulant; however, when bound to a protein carrier the heparin conjugates will become antiplatelet agents by inhibiting collagen-induced platelet aggregation and procoagulant activity, as distinct from soluble heparin. HEP-PG, whether soluble or immobilized to a surface, inhibit platelet deposition on the collagen surface in flowing blood. Mimics of HEP-PG, can be tailored to molecules carrying both antiplatelet and anticoagulant (APAC) properties. These molecules can target the vascular injury site and take residence there. Inhibition of thrombus growth using these APACs under these conditions has been demonstrated in several animal models. Although efficacious for antiplatelet and anticoagulant effects, the bleeding time is shorter with APACs than with unfractionated heparin, suggestive of beneficial efficacy/safety ratio. These strategies may be utilized in drug development, where many vascular injury-related problems can be tackled locally.

\section{Mast Cells As the Origin of Heparin}

Heparin, as used clinically, is one specific member of the glycosaminoglycan (GAG) family, originates from intestinal mucosa and provides a protective anti-inflammatory, antimicrobial, and antithrombotic surface against outer invaders. Heparin was originally derived from liver as a phosphatide, which turned out to possess anticoagulant activity. ${ }^{1}$ Heparin has served for more than 75 years as a source material for modern anticoagulation. The birth of heparin in the United States by the research of Jay MacLean and William Henry Howell has offered a unique and longlasting clinical tool to manage and prevent thrombosis. ${ }^{1}$ Heparin is the preferred anticoagulant to prevent contact activation during certain procedures, such as heart and vascular surgery and plasma exchange. The structural studies for the first clinical use of heparin were performed in 1935 by a Finnish doctor, Erik Jorpes, who had moved in 1919 from Finland to Stockholm Karolinska Institute. ${ }^{2}$ An anecdote of his fanaticism for this material is written in his biography by Runar Beckman: "When his wife was pregnant and the delivery was near some problems arose. Jorpes was working late in the evenings in the laboratory, and when a friend asked him to come home to help his wife he responded that babies are born all the time but heparin is born only once." ${ }^{3}$

The unfractionated heparin (UFH) is isolated from porcine or bovine intestinal mucosa and is used in pharmaceutical manufacturing processes as active product ingredient. It is further chemically or enzymatically degraded to lower molecular weight species, which has been the mainstay of acute anticoagulation needs for several decades.
Issue Theme A Short History of Thrombosis and Hemostasis: Part II (40th Year Celebratory Issue); Guest Editor: Emmanuel J. Favaloro, PhD, FFSc (RCPA).
Copyright $\odot 2014$ by Thieme Medical Publishers, Inc., 333 Seventh Avenue, New York, NY 10001, USA.

Tel: +1(212) 584-4662.
DOI http://dx.doi.org/ 10.1055/s-0034-1395157. ISSN 0094-6176. 
At the time of writing this article, Seminars in Thrombosis $\mathcal{E}$ Hemostasis (STH) contains, according to PubMed, as many as 592 contributions where heparin is mentioned. The oldest entry dates back to 1975 , by Gallus et al, "Thrombolysis with a Combination of Small Doses of Streptokinase and Full Doses of Heparin"). ${ }^{4}$ During the years, many insights into the heparin molecule, its structure and function, as well as laboratory measures and clinical management have been provided by the journal. The history of heparin and STH has shared parallel and complementary routes. The low-molecular-weight heparin, its subcutaneous administration, and even the ultralow molecular species and the antithrombin-binding pentasaccharide and entities without anticoagulant activities have been vividly discussed during the past 40 years. Therefore, it is a great privilege for us to write this article and propose the parent heparin proteoglycan as a model of future drug development.

The GAG surface is typical of all vasculature and organs. The glycocalyx delineates endothelial and platelet surfaces and protects them from simple contact activation. This natural barrier needs to be disrupted in order to initiate coagulation activity, which according to its physiology localizes and limits the action to the site of injury. Under severe coagulation disorders, such as disseminated intravascular coagulation and thrombotic thrombocytopenic purpura, this protective function is lost or altered and generalized thrombosis occurs. For this purpose, the endothelial glycocalyx and the heparan sulfate provide the scaffold to support the potent anticoagulative roles of several molecular interactions, such as antithrombin, tissue factor pathway inhibitor, protein $\mathrm{C}-\mathrm{S}$ system, heparin cofactor II, fibrinolysis, and protection against complement system-mediated activation and fostering regulatory factor $\mathrm{H}$ function.

Mast cells are an integral component of the inflammatory triad, together with neutrophils and lymphocytes. Mast cells reside in tissues, in the peritoneal cavity, in the lungs and vasculature, where they in particular surround the outer surface of vessels, in the adventitial layers. This is the very same site where the strongest tissue factor activity is expressed. The deeper the damage, the more coagulation activity occurs within a given vessel. Thus, the regulatory steps for this activation need to be adopted accordingly. The tissueassociated mast cells upon their activation release heparin and chondroitin proteoglycans (HEP-PG and CS-PG, respectively). HEP-PG are large, on average, $750 \mathrm{kDa}$ structures, containing over 10 heparin chains, of various lengths (likely 20-75 kDa) bound to serine-glycine backbone. This heparin structure also supports the activity of enzymes, such as chymase and tryptase, which both exert anticoagulant properties, such as inhibition of thrombin. ${ }^{5,6}$ Moreover, mast cells contain plasminogen activators, being simultaneously devoid of their regulators of plasminogen-activator inhibitors (PAI-1 and PAI-2), as opposed to all other known cell types. ${ }^{7}$

\section{HEP-PG Are Strong Inhibitors of Platelet Aggregation and Deposition to Collagen}

We isolated serosal mast cells and noted that when activated these release the HEP-PG, which then exhibits a strong inhibi- tory action against platelet aggregation induced by collagen. ${ }^{8}$ UFH has been used as the control for HEP-PG in all these experiments. Also, under high shear rate blood flow over collagen, the platelet deposition is significantly reduced. HEP-PG attenuate platelet-collagen interactions under blood flow via von Willebrand factor (VWF) and platelet glycoprotein (GP)IIb/IIla-mediated mechanisms. When chondroitinasetreated HEP-PG was used, the inhibitory capacity was maintained, whereas heparinase eliminated the platelet inhibitory reactions of HEP-PG. HEP-PG also arrests platelet thrombus growth at the immobilized collagen surface in vitro and vascular injury sites in vivo., ${ }^{9,10}$ The inhibition of platelet deposition and thrombus growth was evidenced both in solution when spiked in whole blood and upon immobilization on the collagen surface. Indeed, when immobilized directly to the collagen surface, platelet deposition was diminished. In these experiments UFH was significantly more potent anticoagulant via antithrombin than HEP-PG, whereas HEP-PG exerted its anticoagulant action via heparin cofactor II (Coenraad Hemker, MD, PhD, and Suzette Beguin, MD, PhD, unpublished data, 1998). In the in vivo experiments, the locally administered HEP-PG at the site of rat femoral artery anastomosis maintained patency while saline and UFH failed to do so. ${ }^{10}$

The concept of mast cell-derived tissue regulation of coagulation was studied in human bleeding time (BT) wounds, where allergen-induced challenge in the BT wound prolonged the BT over the period of follow-up. ${ }^{11}$ The prolongation of BT started at 60 minutes and was maximal at 120 minutes after the allergen challenge in comparison with the histamine control. Also, lesions of cutaneous mastocytoma patients when subjected to BT wound expressed prolonged BT from the injury site.

In a rodent study of vascular anastomosis, the local administration of UFH versus HEP-PG clearly showed that the HEP-PG could maintain the vessel patency, and the 111Indium-labeled-infused platelets deposited less to the anastomosis surface with the exposed collagen and vascular matrix in the presence of HEP-PG than in the presence of UFH. ${ }^{10}$ Rat femoral arteries were cross-sectioned and the anastomosis was created in a regular manner. Before closing the last sutures the inner vessel surface was flushed with a small volume of solution with HEP-PG and the blood flow was allowed to enter the vessel. In - Fig. 1, the uncovered anastomosis surface can be recognized with the visible stitches, whereas in the controls large thrombi were encountered on the vascular anastomosis sites. The HEP-PG has also been shown to carry significant antiproliferative properties in vascular smooth muscle cells, exceeding that of pharmaceutical heparin species. ${ }^{12}$

\section{HEP-PG Mimetics APACs As Dual Antiplatelet and Anticoagulants}

We have constructed semisynthetic molecule complexes which can be tailored to contain a desired amount of heparin chains and they express both anticoagulant and antiplatelet actions, especially against platelet aggregation induced by collagen. The platelet inhibition becomes a highly specific feature, which the 

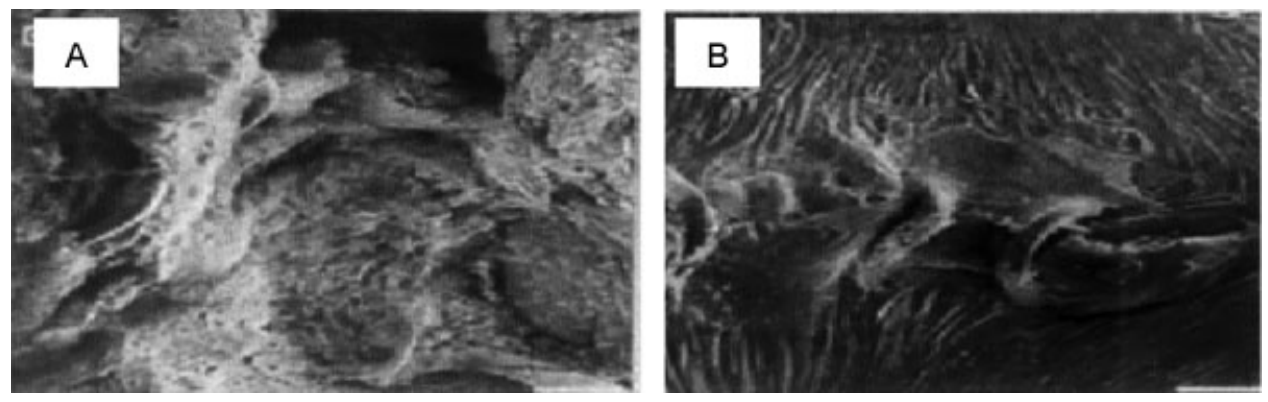

Fig. 1 Scanning electron micrograph of an anastomosis site in a rat femoral artery when either UFH (A) or HEP-PG (B) had been administered locally by flushing (at $100 \mu \mathrm{g} / \mathrm{mL}$ ) with the solution before sacrificing the animal. The anastomosis site was opened for tissue preservation. HEP-PG, heparin proteoglycans; UFH, unfractionated heparin. (Reprinted with permission from Olsson E, Asko-Seljavaara S, Lassila R. Topically administered macromolecular heparin proteoglycans inhibit thrombus growth in microvascular anastomoses. Thromb Haemost 2002;87:245-251.)

soluble unfractionated and low-molecular-weight heparin species do not possess at clinically relevant or even higher concentrations. In contrast, heparin has in fact been shown also to exert some direct activation on platelets as well as the wellknown immunological complications of heparin-induced thrombocytopenia and thrombosis. ${ }^{13-15}$ APACs (antiplatelet and anticoagulant constructs) are conjugates with dual antiplatelet and anticoagulant properties. We will report the antithrombotic effects of APACs in vitro, and in rat vascular anastomosis and baboon thrombosis models in vivo.

\section{Methods}

Agonist-induced (collagen, Nycomed Pharma, Munich Germany; Chrono-Par, Chronolog Corp., Havertown, PA; ristocetin, Helena Biosciences, Gateshaed, United Kingdom; ADP, Sigma-Aldrich, St Louis, MO) platelet aggregation was assessed in citrate anticoagulated blood in platelet-rich plasma (PRP) (Aggram, Helena Biosciences). The anticoagulant effects of APAC and UFH were compared using activated partial thromboplastin time (aPTT), ${ }^{16}$ prothrombin time (PT), thrombin time, anti-FXa activity, fibrinogen, FVIII:C, VWF activity and antigen, and calibrated automated thrombogram (CAT) ${ }^{17,18}$ measurements. PT and aPTT were always measured using Thromborel-S and Actin FSL reagents (both from Siemens Inc., Germany). In CAT, platelet-poor plasma (PPP) was supplemented with $5 \mathrm{pM}$ tissue factor (TF) and PRP with $1 \mathrm{pM}$ TF (PPP reagent and PRP reagent, respectively, Stago, France).

The intravenously administered anticoagulant efficacy and safety of APACs, UFH, and vehicle (saline) were compared in a rat tail BT model. ${ }^{19,20}$ In brief, BT experiments were performed under general anesthesia (2\% isoflurane-02) and study compounds were administered and blood was collected via femoral vein catheter. Before dosing, blood was collected in $3.2 \%$ sodium citrate for aPTT and PT analysis.

APACs, UFH or the vehicle were injected within 30 seconds. At 5 minutes a blood sample was collected to measure aPTT and control the level of anticoagulation. At 10 minutes the tail was cut at $5 \mathrm{~mm}$ from the distal tip with a scalpel and immersed in warm $\left(35 \pm 2^{\circ} \mathrm{C}\right) 0.9 \%$ saline. The BT was recorded until the bleeding had been ceased at least for 5 to 10 seconds.

The antithrombotic efficacy of APACs and UFH in comparison with vehicle was also studied in two well established models of acute thrombosis in baboons. In a modified Folts model an extracorporeal AV-shunt was created between the femoral artery and vein in an anesthetized animal. ${ }^{21,22}$ The blood flow was controlled by an external constrictor placed on the artery and the flow was monitored with a probe. The artery was injured from outside by cross-clamping twice for 10 seconds with a Martin needle holder (Hegar-Baumgartner TC Gold $14 \mathrm{~cm}$ ). All side branches in the proximity of the injury were ligated. The shunt was punctured with a needle (26 G) $1 \mathrm{~cm}$ proximal to the vascular access for injecting a bolus $(4 \mathrm{mg} / \mathrm{mL}$ ) of APAC, UFH or saline. The injury was treated for 3 minutes with the study substance before being exposed to blood flow. Immediately after recovering the baseline blood flow, an external constrictor was placed on the injury and flow was reduced to 30 to $100 \mathrm{~mL} / \mathrm{min}$ (reflecting a stenosis of 90 to 30\%). The accumulation of platelets on the stenosed artery was detected by the reduced blood flow and recorded as cyclic flow reductions (CFR). At $5 \mathrm{~mL} / \mathrm{min}$ the artery was considered occluded and the thrombus was dislodged by releasing the constrictor and flushing with saline. After baseline blood flow was recovered, stenosis was reapplied and the experiment repeated.

In the second baboon model, thrombosis was induced by placing collagen-coated polytetrafluoroethylene grafts $(2 \mathrm{~cm}$, 4-mm lumen) into an externalized arteriovenous shunt. ${ }^{23}$ The thrombogenic collagen surface was treated for $10 \mathrm{mi}-$ nutes with APAC or UFH (both at $4 \mathrm{mg} / \mathrm{mL}$ ). Blood flow was initiated $(100 \mathrm{~mL} / \mathrm{min}$; a shear rate of $265 / \mathrm{s})$ and the deposition of 111-Indium-labeled platelets and fibrin (accumulation of 125-lodine-fibrinogen) was quantified for 60 minutes in an anesthetized animal. Efficacy, distribution, and retention onsite of locally administered 64-Cu-labeled APAC or UFH ( $3 \mathrm{mg} / \mathrm{kg}$ ) were assessed by positron emission tomography (PET) imaging for 50 hours of partially ligated (two loose sutures $1 \mathrm{~cm}$ apart) femoral artery anastomoses in rats.

\section{APAC Inhibits Collagen-Induced Platelet Aggregation with Simultaneous Anticoagulant Efficacy In Vitro}

APAC inhibited platelet aggregation in human PRP induced by collagen and ristocetin (the latter requiring about a 10-fold higher concentration of APAC) in contrast to UFH, but not by 
ADP. The difference between the doses to reach at least $70 \%$ inhibition of platelet aggregation, varied between 3 to $90 \mu \mathrm{g} / \mathrm{mL}$ in collagen-induced platelet aggregation in citrate anticoagulated PRP between the blood donors (-Fig. 2). The mean ED50 dose to inhibit collagen-induced aggregation was approximated to $30 \mu \mathrm{g} / \mathrm{mL}(n=12$; independent donors; inhibition $54 \pm 24 \%$, mean and SD).

The anticoagulant effects of APAC measured by aPTT and thrombin time were comparable or even somewhat better than UFH, while FVIII, fibrinogen, and VWF variables remained unaltered (results not shown) at an equal heparin concentration in vitro. The anticoagulant action of APAC was neutralized by protamine sulfate to a similar manner as with UFH (data not shown).

In the presence of platelets, APAC molecules dose-dependently impaired thrombin generation by initially prolonging the lag time and time to peak (ttpeak), while reducing the peak and endogenous thrombin potential (ETP) both in citrate anticoagulated PPP (supplemented with 5 pM TF and triggered with $\mathrm{Ca}^{2+}$ ) and PRP (supplemented with 1 pM TF and triggered with $\mathrm{Ca}^{2+}$ ). In the representative CAT analysis in PRP (-Fig. 3A-C), the baseline (phosphate buffered saline; PBS) lag time was 9 minutes, ttpeak 20 minutes, peak $58 \mathrm{nM}$, and ETP $965 \mathrm{nM}$. At $0.5 \mu \mathrm{g} / \mathrm{mL}$, UFH prolonged the lag time $45 \%$, ttpeak $48 \%$ and reduced the peak $11 \%$ in comparison with the baseline. At $0.5 \mu \mathrm{g} / \mathrm{mL}$, APAC prolonged the lag time by $70 \%$, ttpeak by $134 \%$, and reduced the peak by $30 \%$ in comparison with the baseline (-Fig. 3A). Overall, APAC prolonged the lag time 1.6-fold, ttpeak 2.8-fold, and reduced the peak 2.7-fold more than UFH. At $1.0 \mu \mathrm{g} / \mathrm{mL}$ UFH prolonged the lag time $75 \%$, ttpeak $159 \%$, and reduced the peak $41 \%$, while in contrast, already at $1.0 \mu \mathrm{g} / \mathrm{mL}$ APAC abolished the thrombin generation ( - Fig. 3B). At $1.5 \mu \mathrm{g} / \mathrm{mL}$, UFH prolonged the lag time $79 \%$, while the ttpeak and peak were immeasurable (-Fig. 3C). ETP was not determined for UFH and APAC due to the significantly delayed thrombin generation at heparin concentrations of 0.5 to $1.5 \mu \mathrm{g} / \mathrm{mL}$.

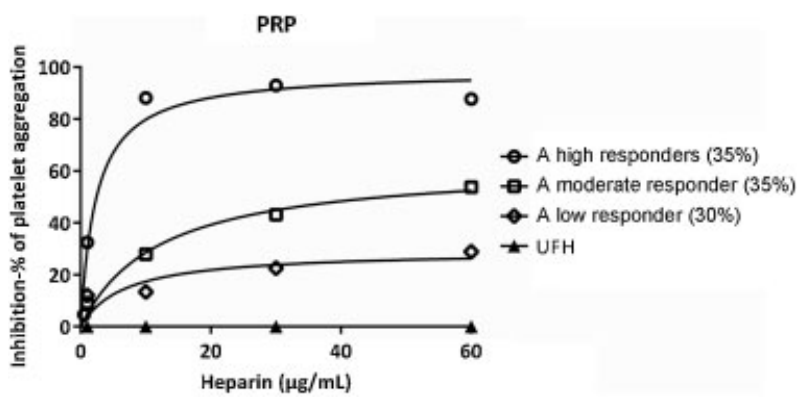

Fig. 2 Inhibition of collagen-induced maximal platelet aggregation in the presence of APAC in citrate anticoagulated PRP with three different donors: A high (open circle), a moderate (open square), and low (open diamond) responder to APAC. The mean inhibition in all three donors in the presence of UFH (black triangle) is shown. Inhibition of the maximal platelet aggregation relative to the vehicle (PBS) is shown as a percentage (\%). APAC, antiplatelet and anticoagulant; PBS, phosphatebuffered saline; PRP, platelet-rich plasma; UFH, unfractionated heparin.
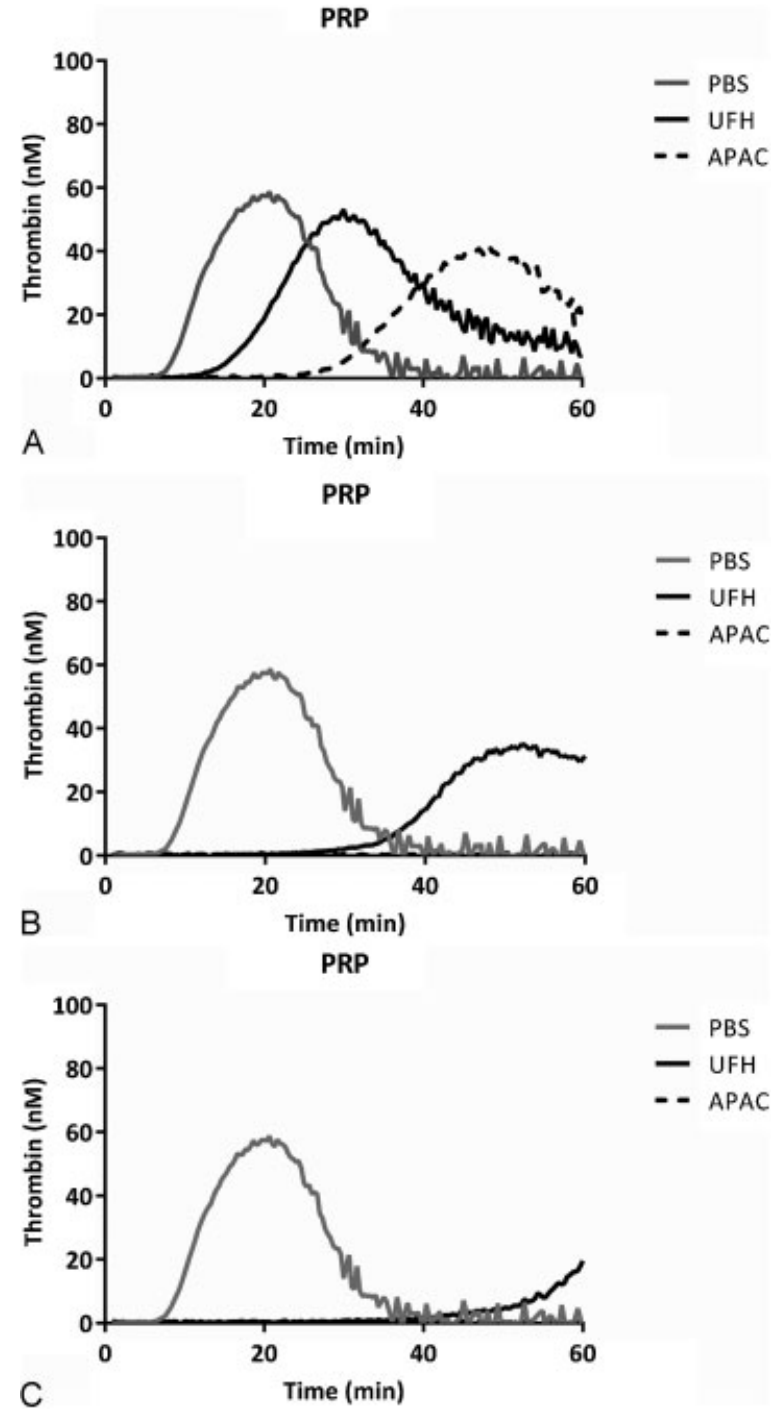

Fig. 3 Reduced thrombin generation in PRP in the presence of APAC compared to UFH using CAT. The thrombograms in the presence of vehicle (PBS) and of (A) $0.5 \mu \mathrm{g} / \mathrm{mL}$, (B) $1.0 \mu \mathrm{g} / \mathrm{mL}$ and (C) $1.5 \mu \mathrm{g} / \mathrm{mL}$ of APAC or UFH in citrate anticoagulated PRP supplemented with $1 \mathrm{pM}$ TF are shown. APAC, antiplatelet and anticoagulant; CAT, calibrated automated thrombogram; PBS, phosphate-buffered saline; PRP, platelet-rich plasma; UFH, unfractionated heparin.

\section{APAC in a Rat Tail BT Model and Acute Models of Thrombosis in Primates}

APAC variables APAC1 and APAC2 (with double coupling efficacy of heparin compared to APAC1), and the UFH prolonged the aPTT and rat tail BT $(n=5-8)$ in comparison with the saline-treated animals. aPTT was prolonged at a dose of $0.26 \mathrm{mg} / \mathrm{kg}$ by eightfold with APAC1 but only doubled with APAC2 over the baseline (-Table 1). At $0.96 \mathrm{mg} / \mathrm{kg}$ aPTT prolonged by 10 -fold with both APACs while UFH had already reached the maximal time measured (180 seconds). At $1.2 \mathrm{mg} / \mathrm{kg}$, also APAC1 induced the maximal prolongation of aPTT while APAC2 clearly (12-fold) prolonged aPTT, which still could be measured. PT was modestly elevated in all 
Table 1 Comparison of APAC1 and APAC2 efficacy on hemostasis in a rat tail bleeding time (BT) model

\begin{tabular}{|l|l|l|l|l|}
\hline $\begin{array}{l}\text { Treatment } \\
\text { mg/kg }\end{array}$ & $\begin{array}{l}\text { BT } \\
\text { Mean (s) } \pm \text { SEM }\end{array}$ & $\boldsymbol{n}$ & $\begin{array}{l}\text { aPTT postdose } \\
\text { Mean (s) } \pm \text { SEM }\end{array}$ & $n$ \\
\hline Saline & $360.00 \pm 54.79^{\mathrm{a}}$ & 6 & $15.02 \pm 0.26$ & 6 \\
\hline UFH 0.96 & $1,949.00 \pm 276.96^{\mathrm{b}}$ & 6 & $180.00 \pm 0.00$ & 5 \\
\hline APAC1 0.26 & $619.17 \pm 63.085^{\mathrm{a}, \mathrm{b}}$ & 6 & $119.14 \pm 37.31$ & 5 \\
\hline APAC1 0.96 & $986.29 \pm 142.04^{\mathrm{a}, \mathrm{b}}$ & 7 & $150.20 \pm 17.56$ & 8 \\
\hline APAC1 1.2 & $2,467.50 \pm 130.58^{\mathrm{b}}$ & 6 & $180.00 \pm 0.00$ & 6 \\
\hline APAC2 0.26 & $803.17 \pm 186.692^{\mathrm{a}, \mathrm{b}}$ & 6 & $27.50 \pm 3.11$ & 6 \\
\hline APAC2 0.96 & $790.67 \pm 128.45^{\mathrm{a}, \mathrm{b}}$ & 6 & $149.10 \pm 15.43$ & 6 \\
\hline APAC2 1.2 & $1,704.17 \pm 264.26^{\mathrm{b}}$ & 6 & $174.83 \pm 5.17$ & 6 \\
\hline
\end{tabular}

Abbreviations: APAC, antiplatelet and anticoagulant; SEM, standard error of mean; UFH, unfractionated heparin.

Note: Saline was used as a negative control and unfractionated heparin (UFH) as the positive control. Samples for activated partial thromboplastin time (aPTT) were collected at 5 minutes after dosing. Detection of bleeding time (BT) was initiated at tail cut 10 minutes after dosing. aPTT was measured up to 180 seconds. The average BT(s) and the aPTT(s) with the standard error of the mean are shown. At $0.96 \mathrm{mg} / \mathrm{kg}$ UFH versus antiplatelet and anticoagulant (APAC) 1 and APAC2, $p$ values were 0.004 and 0.008 , respectively. APACs showed shorter BTs than UFH despite of the relatively equal aPTT prolongation.

a Statistically different from the UFH group, with $p \leq 0.05$ following Student $t$-test.

${ }^{\mathrm{b}}$ Statistically different from the vehicle group.

treatment groups (range , 10-13 seconds) in comparison to the baseline level (9 seconds). Blood cell counts were and remained normal.

At $0.96 \mathrm{mg} / \mathrm{kg}$ UFH (approximately $1.5 \mathrm{IU} / \mathrm{mL}$, a high clinically reachable level) prolonged the tail BT over fivefold the baseline (-Table 1). At 0.26, 0.96, and $1.2 \mathrm{mg} / \mathrm{kg}$, APAC1 prolonged BT by 1.7-, 3-, and 7-folds the baseline, respectively. APAC1 expressed shorter BT from the tail wound than UFH at $0.96 \mathrm{mg} / \mathrm{kg}$. At both 0.26 and $0.96 \mathrm{mg} / \mathrm{kg}$ APAC2 prolonged the BT 2-fold and at $1.2 \mathrm{mg} / \mathrm{kg}$ by 4.7 -fold in comparison with the baseline. At both 0.96 and $1.2 \mathrm{mg} / \mathrm{kg}$ APAC2 exhibited even 20 to 30\% shortened BT than APAC1.

In the acute thrombosis models in baboons APAC and UFH were administered locally on the injury at $4 \mathrm{mg} / \mathrm{mL}$ (-Fig. 4). In the presence of UFH, the artery repeatedly ( 5 CFRs/27 min) occluded at a flow rate of $100 \mathrm{~mL} / \mathrm{min}$ (i.e., stenosed by $30 \%$ ). In contrast, APAC effectively inhibited thrombus formation until the experiment was interrupted, after the follow-up time of 120 minutes. At the end of the experiment, at time point 180 minutes, the artery was restenosed first to $50 \mathrm{~mL} / \mathrm{min}$ (60\% stenosis) and finally to $30 \mathrm{~mL} / \mathrm{min}$ (90\% stenosis), while the local APAC continued to inhibit the occlusion for the selected test periods (10 and 15 minutes, respectively). In the control experiments with UFH and saline repetitive CFRs ensued (results not shown).

In the baboon thrombosis model on the extracorporeal collagen graft, APAC reduced platelet deposition on collagen by $34 \pm 13 \%(n=4, p=0.01)$ in comparison with UFH. The distal thrombus propagation was also diminished by $63 \pm 11 \%(n=4, p=0.19)$ (-Fig. 5). Results with UFH were similar to untreated control values $(n=21)$. Fibrin accumulation was reduced by APAC $(45 \pm 14 \%, n=4)$, but not by UFH $(1.1 \pm 0.1 \%, n=4, p=0.001)$.

In the rat anastomosis model about 10\% APAC attached directly to the vascular application site ( - Fig. 6). Compatible with the strong retention potential and slow degradation, PET detected nondegraded APAC at the anastomotic sites in rats for over 50 hours (up to 120 hours), whereas UFH was undetectable already after 24 hours $(n=2)$. APAC and UFH were cleared via urinary pathway.

\section{Discussion}

APAC molecules show unique dual and localized antiplatelet and anticoagulant action uniformly in several in vitro and in

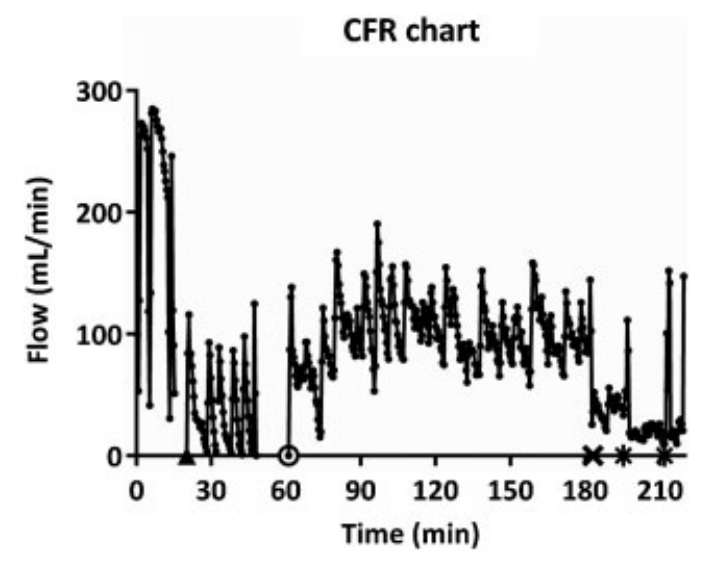

Fig. 4 A chart of CFRs after the local application of UFH and APAC1 (both at $4 \mathrm{mg} / \mathrm{mL} ; 2 \mathrm{mg}$ in total) into the fresh injury site in the modified-Folt model of acute thrombosis in baboons. Immediately after the baseline blood flow returned, the artery was stenosed (30\%) to the flow rate of $100 \mathrm{~mL} / \mathrm{min}$. Repeated occlusion (5 CFRs within 25 minutes) was seen at the injury site treated with UFH (black triangle). In comparison with APAC treatment (open circle) the fresh injury site remained open for the duration of the experiment: First for 120 minutes at the arterial blood flow of $100 \mathrm{~mL} / \mathrm{min}$ (open circle), secondly for 14 minutes with tightened stenosis at the arterial blood flow of $50 \mathrm{~mL} / \mathrm{min}$ (black cross), and finally for 10 and 15 minutes sequential periods at harsh stenosis at blood flow of $30 \mathrm{~mL} / \mathrm{min}$ (black stars). APAC, antiplatelet and anticoagulant; CFR, cyclic flow reduction; UFH, unfractionated heparin. 


\section{At the site of application}

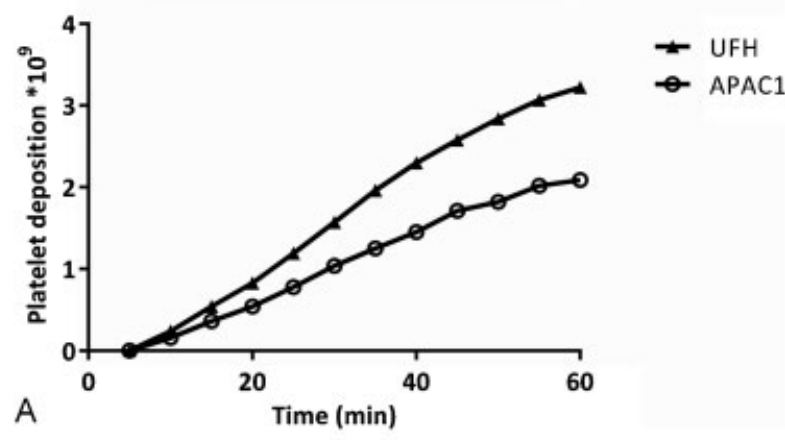

Distally at $10 \mathrm{~cm}$

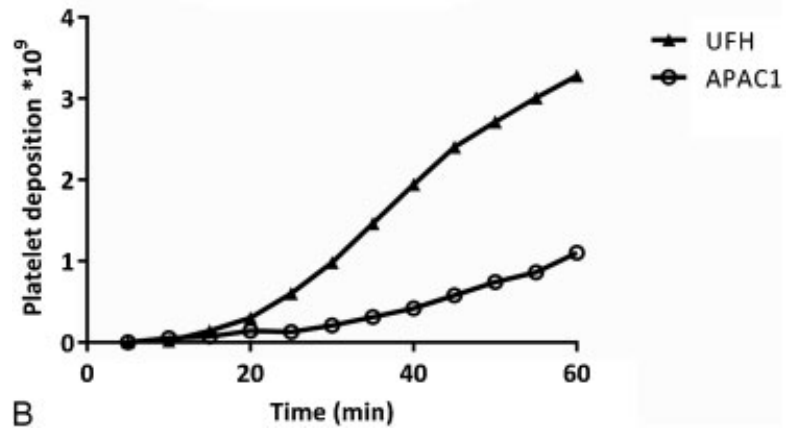

Fig. 5 Comparison of APAC1 and UFH (both at $4 \mathrm{mg} / \mathrm{mL}$ ) in collageninduced thrombus formation in a baboon model $(n=4)$. Reduced platelet deposition was seen in (A) collagen surfaces at the site of application where the platelet on deposition was reduced in the presence of APAC1 by $34 \pm 13 \%$ (SD; $\pm 6 \%$ SEM) in comparison with UFH $(p=0.01)$ and (B) thrombus that propagated $10 \mathrm{~cm}$ distal to the collagen segment where the platelet deposition was reduced at the presence of APAC1 by $63 \pm 11 \%$ (SD; $\pm 5 \%$ SEM) in comparison with UFH $(p=0.19)$. APAC, antiplatelet and anticoagulant; SD, standard deviation; SEM, standard error of mean; UFH, unfractionated heparin.

vivo experiments. In addition to inhibition of platelet aggregation by collagen and anticoagulation (prolongation of aPTT and thrombin time) in human PRP and PPP, in the absence of any systemic anticoagulation, APACs were efficient with local administration in vivo in two baboon models of acute arterial thrombosis and collagen-induced platelet deposition and fibrin formation. APACs may be beneficial under several vascular applications where localized thrombosis continues to be a problem despite the use of current antithrombotic medications. These current agents are systemically administered and also pose a bleeding hazard, which may also be lessened by the local drug administration due to a low concentration but prolonged action. We obviously need to demonstrate that there is no significant leakage to the tissue and that the vascular healing continues in a physiological manner. Our preliminary experiments after 2 weeks of local application support intact healing and vascular structures at the site of anastomosis, which has been treated with our APAC compounds.

In vitro CAT experiments in the presence and absence of platelets in plasma continue to indicate the dual anticoagulant effect and inhibition of platelet-dependent procoagulant action of APACs. The anticoagulant effects of APACs were comparable with UFH at similar dose levels in vitro as previously reported. ${ }^{24}$ In the presence of platelets, APAC impaired thrombin generation initially by prolonging the lag time and ttpeak clearly more than equal doses of UFH. In sharp contrast to UFH, APAC inhibited the collagen-induced platelet aggregation in PRP, but similar to UFH, APAC had no effect on ADP-induced platelet aggregation in PRP.

In vivo $\mathrm{BT}$ experiments in rats showed a systemic aPTT prolongation compatible with UFH, but again in deviation from UFH shortened BT, despite the dual anticoagulant and antiplatelet in vitro action. The BT method of rat tail transection, where the tail is immersed in warm saline, is uniformly used to study the anticoagulant compounds regarding efficacy but more importantly safety. In the BT experiments the baseline results were compatible with the previously reported data, and intravenously administered APACs- and UFH-modified hemostasis as shown by the prolongation of the aPTT and BT. ${ }^{25,26}$ APAC1 and APAC2 induced 50\% less bleeding and prolonged aPTT at least $15 \%$ less in comparison with UFH at the similar dose. APAC1 showed dose-dependent effects on prolonging the $\mathrm{BT}$ at the selected dose range while APAC2 with the doubled coupling level of heparin chains induced less bleeding. APACs may limit simultaneously platelet function by inhibiting collagen-induced platelet aggregation and modifying the intrinsic coagulation pathway and anticoagulants in vivo. Importantly, no significant changes were detected in platelet number or other blood cell count during and after these experiments.

The two in vivo experiments in baboons are encouraging regarding the dual antiplatelet and anticoagulant action. 27,28 In the modified Folt model of acute thrombosis in baboons, APAC applied locally on the crush-injured and stenosed arterial site effectively and consistently inhibited thrombosis in the artery under the moderate shear stress, with the flow rate of $100 \mathrm{~mL} / \mathrm{min}$ and stenosis of $30 \%$ until the experiment was interrupted after 120 minutes. When APAC was addressed with the short exposure of sequentially increased stenosis from 60 to $90 \%$ the patency of the artery was further maintained. In addition, in the extracorporeal collagen graft model, APAC inhibited both thrombosis and fibrin accumulation about by $50 \%$ over UFH in the 60 -minute experiment in baboons. The APAC potency was further emphasized with the distally propagated thrombus over the effect of heparin.

APAC has potential to target itself at the vascular injury site. The local administration and residence of APAC on the femoral artery anastomosis was confirmed with the PET scan in rats where the retention time of copper-labeled APAC was at least twice the time of UFH. This finding refers to the capacity of APACs to target at the injury site and to be maintained there for a significant period of time to inhibit unwanted thrombotic response. As the vascular injury triggers the inflammatory cellular triad of the leukocytes, lymphocytes, and mast cells with their heparin proteoglycans released to the tissue, the local heparin reaction is a native physiological defense mechanism.

Overall, APAC dually inhibited collagen- and ristocetininduced platelet aggregation in human blood, with anticoagulant properties that were at least comparable with UFH in 


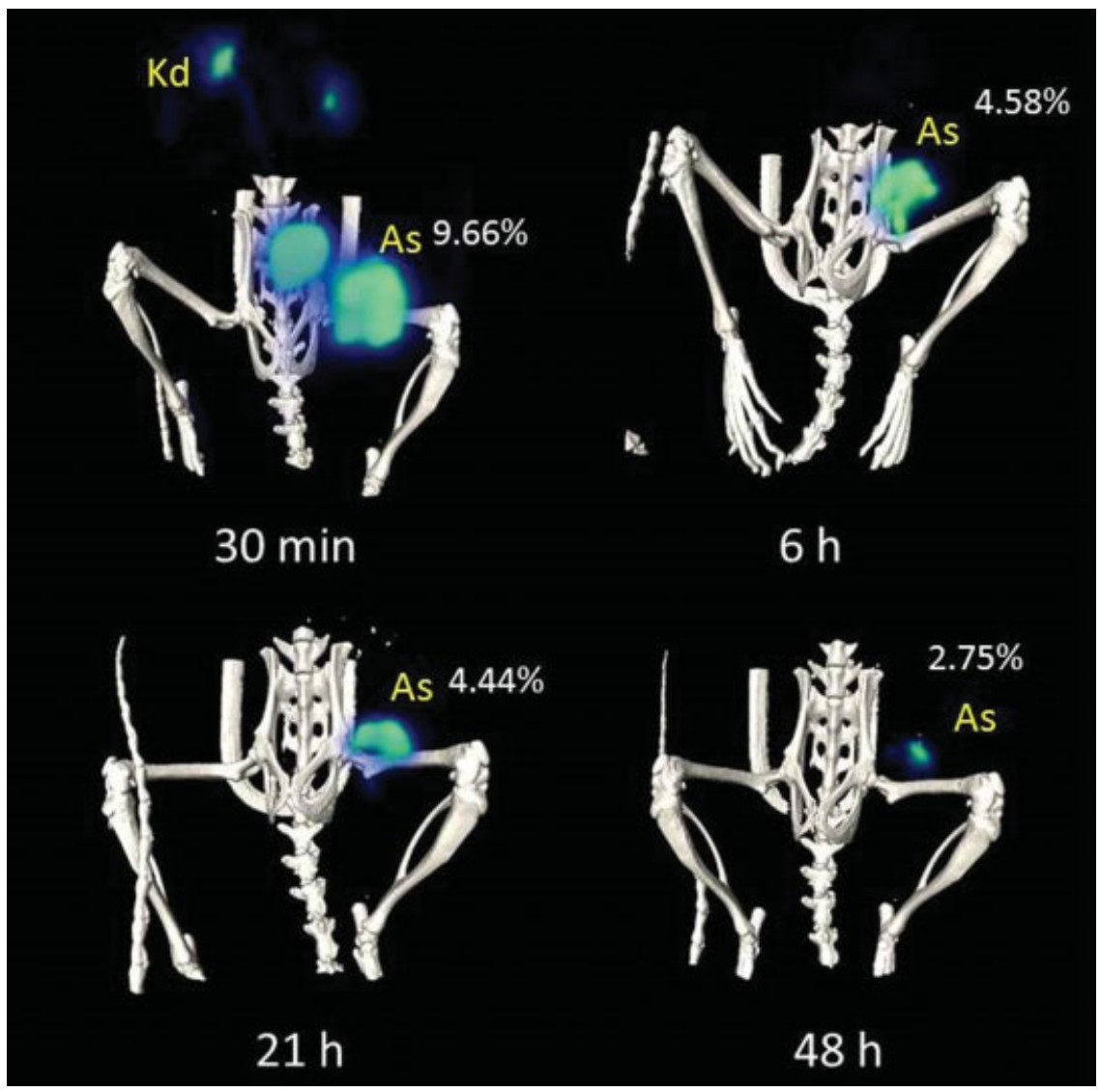

Fig. 6 An example of PET/CT images of the rat at 30 minutes, 6, 21, and 48 hours time points following the local administration of 64-CopperAPAC2 at the site of anastomosis. Remaining radioactivity is shown at the As. PET acquisition was performed on a Triumph PET/CT scanner (Gamma Medica-Ideals, Nortridge, CA). The radioactivity remaining at the As and in the organs was quantified using three-dimensional ROI. Quantitative values at the As are expressed as the percentage-radioactivity relative to the first measurement and corrected for the physical decay of the radiolabel. APAC, antiplatelet and anticoagulant; As, application site; $\mathrm{CT}$, computed tomography; PET, positron emission tomography; ROI, regions of interest.

plasma. In the absence of systemic antithrombotic therapy, treatment of a highly thrombogenic vascular and collagen surfaces with APAC arrested in vivo thrombus formation in two models of acute thrombosis in baboons. Locally administered APAC alone, with its dual antiplatelet and anticoagulant effects, may limit the growth of thrombus and prevent thrombo-occlusions in various applications. The encouraging data in the BT model would support even the systemic administration of APAC. However, the local application and administration to thrombotic sites of vascular injury would represent a novel modality to treat arterial thrombosis in association with interventions and risk of acute thrombosis. This model of antithrombotic tissue action would be compatible with nature's GAGs, including large heparin conjugates derived from mast cells.

\section{Acknowledgments}

We thank the following collaborators: Marja Lemponen, $\mathrm{HUCH}$, Helsinki, Finland; Jan Roodt, PhD, and Sep Lamprecht, Bloemfontein, South Africa; Ulla Marzec, MSc, and Stephen Hanson, PhD, formerly from Oregon Health \&
Sciences University, Portland, OR; Charles Laurent, PhD, and Dany Salvail, IPST, Sherbrook, Canada; and Gijs Van Dedem, PhD, formerly from Organon, Oss, The Netherlands.

\section{References}

1 Wardrop D, Keeling D. The story of the discovery of heparin and warfarin. Br J Haematol 2008;141(6):757-763

2 Jorpes E. Heparin in the treatment of thrombosis. An account of its chemistry, physiology and application in medicine. 2nd ed. New York, NY: Oxford University Press; 1946

3 Bäckman R. Erik Jorpes, Kökar-Moskva-Stockholm. 2nd ed. Helsinki, Finland: Söderström; 1985

4 Gallus AS, Hirsch J, Cade JF, Turpie AG, Walker IR, Gent M. Thrombolysis with a combination of small doses of streptokinase and full doses of heparin. Semin Thromb Hemost 1975;2(1):14-32

5 Tchougounova E, Pejler G. Regulation of extravascular coagulation and fibrinolysis by heparin-dependent mast cell chymase. FASEB J 2001;15(14):2763-2765

6 Bode W. The structure of thrombin: a janus-headed proteinase. Semin Thromb Hemost 2006;32(Suppl 1):16-31

7 Valent P, Baghestanian M, Bankl HC, et al. New aspects in thrombosis research: possible role of mast cells as profibrinolytic and antithrombotic cells. Thromb Haemost 2002;87(5):786-790 
8 Lassila R, Lindstedt K, Kovanen PT. Native macromolecular heparin proteoglycans exocytosed from stimulated rat serosal mast cells strongly inhibit platelet-collagen interactions. Arterioscler Thromb Vasc Biol 1997;17(12):3578-3587

9 Kauhanen P, Kovanen PT, Lassila R. Coimmobilized native macromolecular heparin proteoglycans strongly inhibit platelet-collagen interactions in flowing blood. Arterioscler Thromb Vasc Biol 2000; 20(11):E113-E119

10 Olsson E, Asko-Seljavaara S, Lassila R. Topically administered macromolecular heparin proteoglycans inhibit thrombus growth in microvascular anastomoses. Thromb Haemost 2002;87(2):245-251

11 Kauhanen P, Kovanen PT, Reunala T, Lassila R. Effects of skin mast cells on bleeding time and coagulation activation at the site of platelet plug formation. Thromb Haemost 1998;79(4):843-847

12 Wang Y, Kovanen PT. Heparin proteoglycans released from rat serosal mast cells inhibit proliferation of rat aortic smooth muscle cells in culture. Circ Res 1999;84(1):74-83

13 Gao C, Boylan B, Fang J, Wilcox DA, Newman DK, Newman PJ. Heparin promotes platelet responsiveness by potentiating $\alpha$ IIb $\beta 3$ mediated outside-in signaling. Blood 2011;117(18):4946-4952

14 Warkentin TE, Greinacher A, Gruel Y, Aster RH, Chong BH; scientific and standardization committee of the international society on thrombosis and haemostasis. Laboratory testing for heparin-induced thrombocytopenia: a conceptual framework and implications for diagnosis. J Thromb Haemost 2011;9(12): 2498-2500

15 Lassila R, Antovic JP, Armstrong E, et al. Practical viewpoints on the diagnosis and management of heparin-induced thrombocytopenia. Semin Thromb Hemost 2011;37(3):328-336

16 Lippi G, Favaloro EJ. Activated partial thromboplastin time: new tricks for an old dogma. Semin Thromb Hemost 2008;34(7):604-611

17 Al Dieri R, de Laat B, Hemker HC. Thrombin generation: what have we learned? Blood Rev 2012;26(5):197-203

18 Adams M. Assessment of thrombin generation: useful or hype? Semin Thromb Hemost 2009;35(1):104-110
19 Dejana E, Callioni A, Quintana A, de Gaetano G. Bleeding time in laboratory animals. II - A comparison of different assay conditions in rats. Thromb Res 1979;15(1-2):191-197

20 Dejana E, Callioni A, Quintana A. de Gaetano G. Bleeding time in laboratory animals. III - Do tail bleeding times in rats only measure a platelet defect? (the aspirin puzzle). Thromb Res 1979; 15:199-207

21 Folts J. An in vivo model of experimental arterial stenosis, intimal damage, and periodic thrombosis. Circulation 1991;83(6, Suppl) IV3-IV14

22 Fontayne A, Meiring M, Lamprecht S, et al. The humanized antiglycoprotein Ib monoclonal antibody h6B4-Fab is a potent and safe antithrombotic in a high shear arterial thrombosis model in baboons. Thromb Haemost 2008;100(4):670-677

23 Hanson SR, Kotze HF, Savage B, Harker LA. Platelet interactions with Dacron vascular grafts. A model of acute thrombosis in baboons. Arteriosclerosis 1985;5(6):595-603

24 Bonar RA, Favaloro EJ, Marsden K. External quality assurance for heparin monitoring. Semin Thromb Hemost 2012;38(6):632-639

25 Morishima Y, Honda Y, Kamisato C, Shibano T. Comparison of antithrombotic and hemorrhagic effects of edoxaban, a novel factor Xa inhibitor, with unfractionated heparin, dalteparin, lepirudin and warfarin in rats. Thromb Res 2013;132(2):234-239

26 Takahashi S, Hirai N, Shirai M, Ito K, Asai F. Comparison of the blood coagulation profiles of ferrets and rats. J Vet Med Sci 2011;73(7): 953-956

27 Lassila R, Badimon JJ, Vallabhajosula S, Badimon L. Dynamic monitoring of platelet deposition on severely damaged vessel wall in flowing blood. Effects of different stenoses on thrombus growth. Arteriosclerosis 1990;10(2):306-315

28 Badimon L, Badimon JJ, Lassila R, Heras M, Chesebro JH, Fuster V. Thrombin regulation of platelet interaction with damaged vessel wall and isolated collagen type I at arterial flow conditions: effects of hirudins, heparin and calcium chelation. Blood 1991; $78: 423-434$ 